\title{
In Situ Observations of the Growth Mode of Vacuum-Deposited $\alpha$-Sexithiophene
}

\author{
T. L. Derrien,* A. E. Lauritzen, P. Kaienburg, J. F. M. Hardigree, C. Nicklin, and M. Riede* \\ Cite This: J. Phys. Chem. C 2020, 124, 11863-11869 \\ Read Online
}

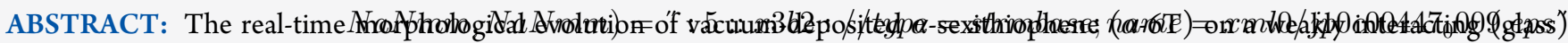
substrate at ambient temperature is reported. In situ grazing-incidence small-angle X-ray scattering (GISAXS) enabled the observation of nanoscale aggregates, while in situ grazing-incidence wide-angle scattering (GIWAXS) allowed the study of the molecular-scale morphology. The in situ GISAXS measurements revealed that the $\alpha-6 \mathrm{~T}$ growth proceeds via a Stranski-Krastanov mode, whereby 2-4 complete monolayers are deposited, followed by subsequent layers formed via island growth. In situ GIWAXS also showed the evolution of the polymorph composition during the thin-film growth. Initially, the disordered $\beta$-phase and the lowtemperature (LT)-phase are deposited in nearly equal proportion until a thickness of $8 \mathrm{~nm}$, whereby the LT-phase begins to dominate until a final $\alpha$-6T thickness of $50 \mathrm{~nm}$ where the scattering intensity of the LT-phase is more than double that of the $\beta$ phase. The change in the polymorph composition coincided with an increase in the LT-phase $d$-spacing, indicating a lattice strain relief as the thin film moves from surface to bulk-mediated growth. The GISAXS findings were confirmed through direct imaging using ex situ atomic force microscopy (AFM) at various thicknesses, revealing the existence of both initial the initial and intermediate monolayers and final island morphologies. The findings reveal the real-time morphological evolution of $\alpha$-6T across both the molecular scale and the nanoscale and highlight the role of strain in polymorph growth. Due to the importance of the thin-film microstructure in device performance, it is expected that these results will aid in the development of structure-property relationships necessary to realize the full potential of organic electronics.

\section{INTRODUCTION}

Small-molecule organic semiconductors (OSCs) have emerged as interesting materials for a variety of applications including organic field-effect transistors (OFETs), ${ }^{1,2}$ organic lightemitting diodes (OLEDs), ${ }^{3}$ and organic photovoltaics (OPVs). ${ }^{4-6}$ In addition to low-cost and environmentallyfriendly processing techniques, the diverse range of molecules available allow tailored structures to be created that can optimize a variety of physical parameters. ${ }^{7,8}$ However, OPV and OFET devices are still outperformed by their inorganic counterparts, in part due to the microstructural complexity of OSC thin films. Such thin-film morphologies are typically amorphous or polycrystalline with several crystalline polymorph components affecting numerous factors, such as crystalline orientation, domain size, and purity, that influence the optical and electronic properties. ${ }^{9-16}$ This complicates theoretical predictions of performance and necessitates detailed studies across multiple length scales, targeting both bulk and interfaces to understand and engineer the structures that influence the properties and realize the potential of OSCs.

$\alpha$-Sexithiophene $(\alpha-6 \mathrm{~T})$ is an OSC material that has been extensively studied for use in OLEDs, ${ }^{17}$ OFETs, ${ }^{18}$ and $\mathrm{OPVs}^{19-21}$ due to its combination of high carrier mobility and appropriate optical gap. Several polymorphs of $\alpha-6 \mathrm{~T}$ have been shown to exist in thin films. ${ }^{22-26}$ The selection of the appropriate polymorph or combination of polymorphs is crucial to device performance, as each polymorph displays distinct properties. For example, the high-temperature (HT) and low-temperature (LT) phases of $\alpha-6 \mathrm{~T}$ show differing absorption strengths (under normal incidence). ${ }^{27,28}$ While the HT and the LT phases exist both in bulk and thin films, another $\alpha-6 \mathrm{~T}$ polymorph is the kinetically favored and disordered $\beta$-phase, which is unique to thin films. ${ }^{24,29} \alpha-6 \mathrm{~T}$ polymorph control in thin films has been shown to be dependent on several parameters such as illumination conditions, ${ }^{27}$ substrate temperature, ${ }^{26}$ deposition rate, ${ }^{30}$ postdeposition annealing, ${ }^{31}$ or vacuum incubation. ${ }^{19}$

Here, we used several methods to characterize the real-time growth of $\alpha-6 \mathrm{~T}$ on a commercially relevant glass substrate to characterize the growth mode across several length scales from several angstroms to $\approx 200 \mathrm{~nm}$. In situ grazing-incidence smallangle X-ray scattering (GISAXS) was used to characterize the nanoscale structure of the growing films, while in situ grazingincidence wide-angle X-ray scattering (GIWAXS) was used to characterize the molecular- scale microstructure. The morphologies observed via X-ray scattering were confirmed through direct surface topography imaging by atomic force microscopy (AFM), revealing a Stanski-Krastanov growth mode. These observations of both the final and intermediate $\alpha$ -

Received: January 16, 2020

Revised: $\quad$ May 9, 2020

Published: May 12, 2020 
6T growth stages provide important insight into the structural control of OSC thin films.

\section{METHODS}

Grazing-Incidence Small-Angle X-ray Scattering (GISAXS). GISAXS measurements used the Diamond Light Source surface diffraction beamline (I07). In situ experiments were performed using the purpose-built MINERVA sample chamber $^{32}$ that consists of dual low-temperature thermal evaporation sources whose deposition rates are monitored by quartz crystal microbalances (QCMs). Prior to $\alpha-6 \mathrm{~T}$ evaporation, the Corning Eagle XG substrates (manufactured with an root mean square (RMS) of $5 \AA$, low roughness confirmed by the lack of discernible diffuse scattering) were cleaned in an ultrasonic bath for 10 min using 2.5\% Hellmanex (non-etching) solution, deionized water, acetone, and finally isopropanol. The cleaned substrate was mounted, and the chamber was pumped down until a base pressure of $10^{-7} \mathrm{mbar}$ was achieved. $\alpha-6 \mathrm{~T}$ was deposited onto the substrate at a rate of $0.3-0.45 \AA / \mathrm{s}$, as monitored by the QCMs, which were calibrated using spectroscopic ellipsometry using film thicknesses of 5, 10, and $50 \mathrm{~nm}$. In situ GISAXS measurements were recorded every $10 \mathrm{~s}$ using an exposure time of $5 \mathrm{~s}$ with an X-ray beam energy of $10.0 \mathrm{keV}$ and an incidence angle of $0.1^{\circ}$ (below the critical angle of the glass substrate $\alpha_{\mathrm{c}} \approx 0.14^{\circ}$ ). No beam damage was detected for thick films, as verified by comparing the images of the X-ray illuminated section with an off-beam section after the deposition. Images were collected using a Pilatus $2 \mathrm{M}$ detector at a sample to detector distance of $3.02 \mathrm{~m}$ that was calibrated using a silver behenate standard (AgBeh). Data reduction was performed in DAWN. ${ }^{33}$

Grazing-Incidence Wide-Angle X-ray Scattering (GIWAXS). In situ GIWAXS measurements were recorded using the previously described MINERVA chamber and deposition conditions on glass substrates with a beam energy of $20.0 \mathrm{keV}$ and an incidence angle of $0.05^{\circ}\left(\alpha_{\mathrm{c}} \approx 0.07^{\circ}\right)$. Images with a $1 \mathrm{~s}$ exposure time were collected every $10 \mathrm{~s}$ using a Pilatus $2 \mathrm{M}$ detector at a sample to detector distance of $41.8 \mathrm{~cm}$ that had been calibrated using AgBeh.

Atomic Force Microscopy (AFM). The AFM images were collected using a Veeco Multimode $\mathrm{V}$ in tapping mode mounted with Bruker SCANASYST-AIR-HR tips. A scanning rate of $0.5 \mathrm{~Hz}$ was used, and the images were analyzed using Gwyddion. ${ }^{34}$ The samples were prepared in the same manner as the in situ samples, but the sample shutter was closed at QCM readings corresponding to $2.5,3.5,4.5$, and $5.5 \mathrm{~nm}$.

\section{RESULTS}

To obtain a complete understanding of the growth mode of $\alpha$ $6 \mathrm{~T}$, we employed several characterization methods capable of probing the thin-film microstructure across broad length scales. In situ GISAXS was used to track the real-time morphology of nanoscale aggregates, and these findings were confirmed via AFM imaging. The evolution of the molecular structure of the $\alpha-6 \mathrm{~T}$ film growth was then monitored in real-time via in situ GIWAXS, which enabled the tracking of the polymorph composition during growth.

Selected detector images from the in situ GISAXS are shown in Figure 1A. The images are taken from time points corresponding to multiples of approximately 0.7-1.3 monolayers (MLs). The number of MLs deposited was calculated using the recorded QCM thicknesses and assuming an LT-
A

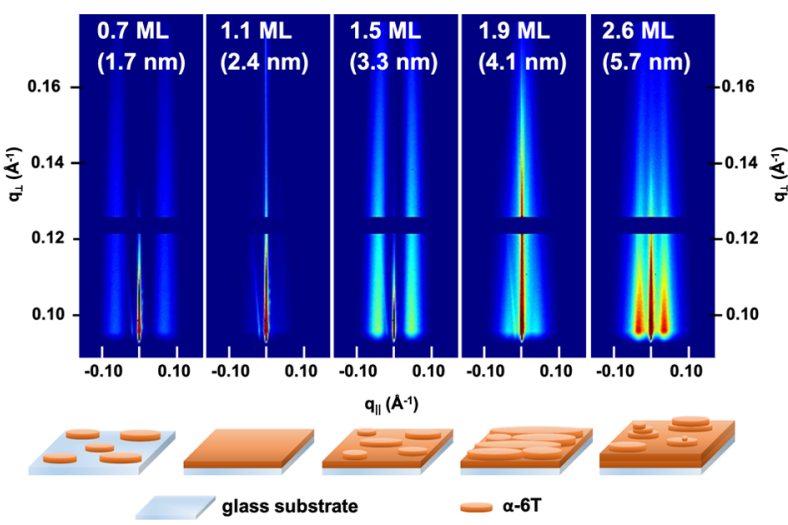

Figure 1. (A) Two-dimensional (2D) in situ GISAXS images obtained at various $\alpha-6 \mathrm{~T}$ ML coverages showing the variation in scattering during the course of the deposition. At $0.7 \mathrm{ML}(1.7 \mathrm{~nm}), 1.5 \mathrm{ML}(3.3$ $\mathrm{nm})$, and 2.6 ML (5.7 nm) coverages, strong scattering peaks are observed, while at $1.1 \mathrm{ML}(2.4 \mathrm{~nm})$ and $1.9 \mathrm{ML}(4.1 \mathrm{~nm})$ coverages, the peaks disappear. (B) Schematic of the growth mode elucidated from the detector images.

phase structure $(2.2 \mathrm{~nm}) .^{24}$ This assumption leads to only approximate ML multiples, due to variation in the $\alpha-6 \mathrm{~T}$ polymorph composition, but is convenient for discussing the resulting small-angle scattering. From these detector images, it can be seen that off-specular scattering is strongest at incomplete $\mathrm{ML}$ multiples (Figure $1 \mathrm{~A} ; 0.7,1.5$, and 2.6 MLs) and weakest at complete or near-complete MLs (Figure 1A; 1.1 and 1.9 MLs). This is a result of strong scattering from island-like morphologies for unfilled MLs and weak scattering from the smooth, complete, ML surface ${ }^{3535}$ (Figure 1B). Such strong oscillations in the scattering intensity only occurred during these early stages of the $\alpha-6 \mathrm{~T}$ deposition. This can be seen in the real-time intensity variation of the scattering in the in-plane direction shown in Figure 2 and in a video of the detector images of the full $50 \mathrm{~nm}$ thin-film deposition (available in the electronic Supporting Information). The data in Figure 2, composed of stacked linecuts at $q_{\perp}=0.095$ $\AA^{-1}$, are indicative of the changing morphology of the growing $\alpha-6 \mathrm{~T}$ film. The appearance and disappearance of the offspecular scattering can be clearly seen in the early time points corresponding to thicknesses of up to 2.6 MLs. The absolute peak intensity was calculated by fitting a Lorentizian shape on a Gaussian background in the linecut data and was plotted against film thickness, as shown in Figure 3A. The first two minima indicated by the dashed lines correspond to the nearly complete monolayer coverage shown in Figure 1, at 1.1 and 1.9 ML coverage. Upon further $\alpha-6 \mathrm{~T}$ deposition, or after 2 MLs have been deposited, the degree of oscillation in the offspecular scattering intensity is reduced and completely absent after $4 \mathrm{MLs}(8.4 \mathrm{~nm})$ corresponding to a loss of the layerwise growth and the presence of $\alpha-6 \mathrm{~T}$ islands.

In addition to the intensity variations, Figure 2 shows the narrowing and broadening of the peaks occurring during these oscillations. Shifts in the peak positions (dashed lines in Figure 2A) are due to the varying correlation length resulting from the changing separation of the growing islands. This disparity can be clearly seen in the sample linecuts and fits for the peaks corresponding to 0.7 and 1.5 MLs shown in Figure 2B, which highlight the variations seen from the first two scattering peaks seen in the time series. The peak positions from the fits were used to calculate the characteristic length scale of the average 
A

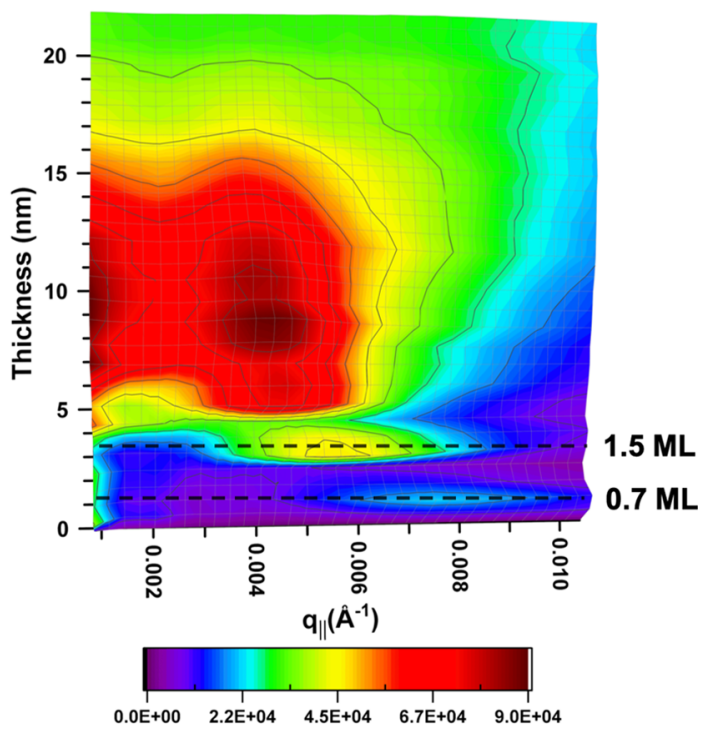

B

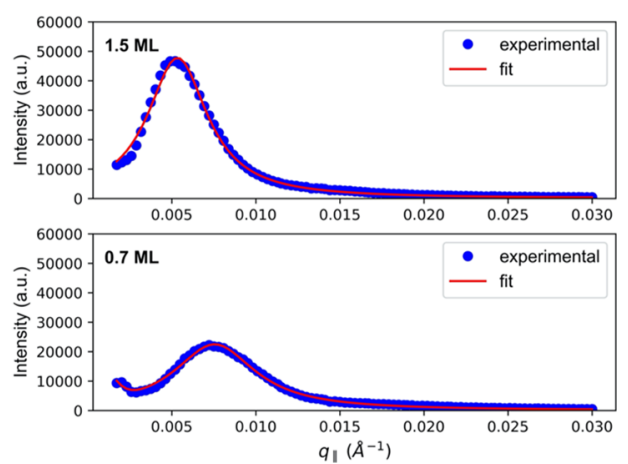

Figure 2. Real-time GISAXS data of $\alpha-6 \mathrm{~T}$ deposited on glass at room temperature. (A) Surface plot of real-time $\alpha-6 \mathrm{~T}$ GISAXS scans measured up to $20 \mathrm{~nm}$ thickness showing variation in off-specular scattering intensity and peak positions. The surface plot is a composite of linecuts taken at $q_{\perp}=0.095 \AA^{-1}$. The peaks indicated with dashed lines occur between the deposition of the first (0.7 ML) and second monolayers (1.5 ML), as shown in Figure 1A. The linecuts taken from the detector images for these peaks are shown in (B), along with the corresponding fits.

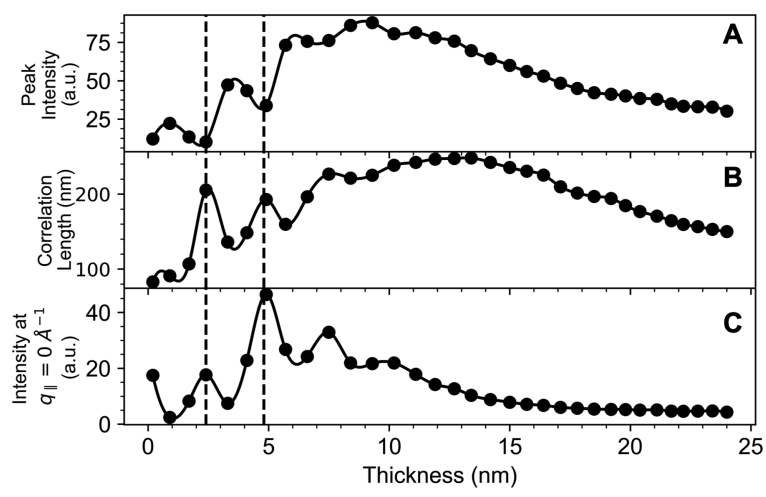

Figure 3. Evolution of the (A) peak scattering intensity, (B) correlation length, and (C) intensity at $q_{\|}=0 \AA^{-1}$ (calculated from a $5 \times 5$ pixel region centered at $q_{\perp}=0.11 \mathrm{~A}^{-1}$ ) of the $\alpha-6 \mathrm{~T}$ film during growth as measured by GISAXS. Dashed lines correspond to the deposition of one and two monolayers. separation of the islands, or the correlation length $\left(L_{\mathrm{c}}\right)$, via $L_{\mathrm{c}}=$ $2 \pi / q_{\|}$, where $q_{\|}$is the peak position in q-space (as confirmed via simulation using BornAgain, ${ }^{36}$ Figure S1). The correlation length of the growing islands is plotted in Figure 3B, which indicates that the islands formed on top of the first monolayer grow with a larger correlation length than those grown on the glass substrate. The correlation length is significantly increased near complete ML coverage. This can be attributed to the fact that near complete ML coverage, the scattering signal is the result of the gaps in the film, not the island separation. The diffuse scattering close to the specular beam $\left(\right.$ at $\left.q_{\|}=0 \AA^{-1}\right)$, calculated from a $5 \times 5\left(0.0015 \AA^{-1} \times 0.015 \AA^{-1}\right)$ pixel region centered at $q_{\perp}=0.11 \AA^{-1}$, was also used to monitor the progression of the film growth (Figure $3 \mathrm{C}$ ), revealing a period of layerwise oscillation. The oscillation in the peak intensity, correlation length, and diffuse scattering near the specular beam were all reduced after 2 MLs were deposited and absent after $4 \mathrm{MLs}$, indicating that after this point, the growth of the $\alpha-6 \mathrm{~T}$ film proceeds via the formation of adsorbate islands. The decreased correlation length observed indicates that in the latter stages of the film growth the islands formed were more closely spaced together. Collectively, the GISAXS data show that $\alpha-6 \mathrm{~T}$ growth by vacuum deposition on glass proceeds via a Stranski-Krastanov $(\mathrm{SK})^{37}$ growth mode where beyond a critical wetting layer thickness of two monolayers, $\alpha-6 \mathrm{~T}$ deposition progresses via island growth (Figure 1B). The observation of SK growth, which depends on lattice interactions (strain) between the surface and the adsorbate, demonstrates the templating potential of the thin-film substrate. Though SK growth modes for $\alpha-6 \mathrm{~T}$ have been observed in situ on metal substrates using photoelectron emission microscopy (PEEM) and reflectance difference spectroscopy (RDS), ${ }^{38,39}$ they have never been expressly captured on weakly interacting, device-relevant substrates such as the glass substrates used here. Furthermore, the in situ GISAXS employed here enabled the calculation of morphological parameters, with $\mathrm{nm}$ resolution, of the growing film not accessible with other techniques.

Direct imaging of the various growth stages via AFM of ex situ-prepared samples was consistent with the analysis of the GISAXS data. We were able to image the early stages of $\alpha-6 \mathrm{~T}$ growth by stopping the deposition at thicknesses of $2.5 \mathrm{~nm}$ (Figure 4A), $3.5 \mathrm{~nm}$ (Figure 4B), $4.5 \mathrm{~nm}$ (Figure 4C), and 5.5 $\mathrm{nm}$ (Figure 4D), as measured by QCM. Additionally, Figure $4 \mathrm{E}$ shows the surface structure of the $50 \mathrm{~nm} \alpha-6 \mathrm{~T}$ film, where the island morphology is clearly visible. Watershed analysis of the islands of the $50 \mathrm{~nm}$-thick film indicated a mean island size of $53 \pm 15 \mathrm{~nm}$. The size of the islands was also estimated using the off-specular GISAXS peaks according to the Scherrer equation, ${ }^{40} D_{h k l}=2 \pi \mathrm{K} / \mathrm{FWHM}$, where $D_{h k l}$ is the average grain size, taken here as the size of the islands, and $K$ is a shape constant, 0.9 for spherical grains. Due to instrumental broadening and detector resolution considerations, Scherrer analysis can be used to calculate a lower limit grain size. ${ }^{41}$ For the $50 \mathrm{~nm}$-thick film, Scherrer analysis returned a lower limit grain size of $41 \mathrm{~nm}$, which is within the standard deviation of the AFM measurement. The image series shows the progression from near monolayer coverage (Figure 4A), to island formation (Figure 4B), growth (Figure 4C), coalescence (Figure 4D), and repeated island formation (Figure 4E) of the $\alpha-6 \mathrm{~T}$ film. This was also observed through the increase of RMS roughness, as calculated from the AFM images (Table 1), where at thicknesses below 2 MLs $(2.5,3.5$, and $4.5 \mathrm{~nm})$, the 

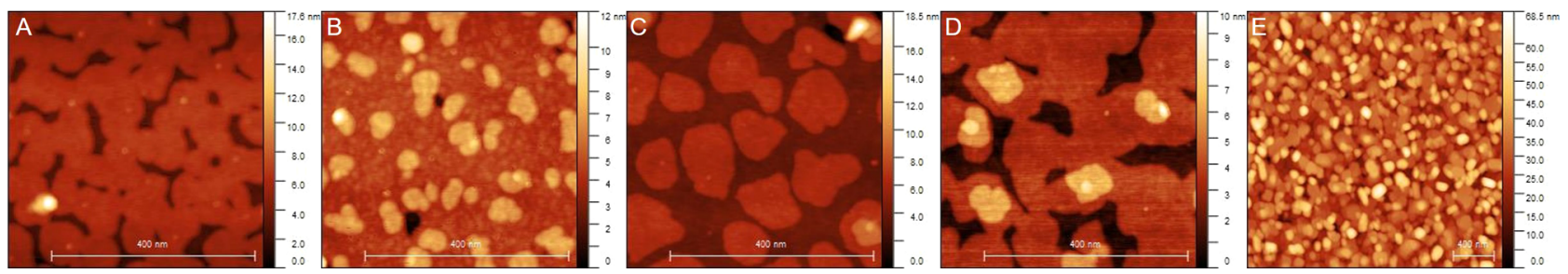

Figure 4. AFM images of vacuum-deposited $\alpha-6 \mathrm{~T}$ films at $(\mathrm{A}) 2.5$ ( $\approx 1.1 \mathrm{ML}),(\mathrm{B}) 3.5(\approx 1.6 \mathrm{ML}),(\mathrm{C}) 4.5(\approx 2.0 \mathrm{ML}),(\mathrm{D}) 5.5(\approx 2.5 \mathrm{ML})$, and (E) $50 \mathrm{~nm}$ thickness.

Table 1. RMS Roughness and Correlation Length Calculated from Figure 4

\begin{tabular}{ccc} 
sample thickness $(\mathrm{nm})$ & RMS roughness $(\mathrm{nm})$ & correlation length $(\mathrm{nm})$ \\
2.5 & 1.2 & 157 \\
3.5 & 1.2 & 132 \\
4.5 & 1.5 & 142 \\
5.5 & 3.2 & 150 \\
50 & 8.2 & 98 \\
\hline
\end{tabular}

RMS roughness remains approximately constant, after which it quickly increases as a result of island formation (5.5 and 50 $\mathrm{nm}$ ). The ex situ AFM observations further confirm the SK growth mode observed in situ above. Additional image analysis was conducted to extract an approximate correlation length of the islands (Figure S2). The results shown in Table 1 correspond with the correlation lengths extracted from the GISAXS data.

The crystalline structure of the growing $\alpha-6 \mathrm{~T}$ film was monitored by real-time in situ GIWAXS measurements. By performing these time-resolved measurements under the same conditions as the above GISAXS experiments, we were able to track the evolution of the different $\alpha-6 \mathrm{~T}$ polymorphs throughout the deposition process. Figure 5A, composed of a series of linecuts taken from the in-plane direction, shows the evolution of the $\beta$ - and the LT-phase peaks during the film growth. The heatmap clearly shows the inverse relation between the intensity of the $\beta$ and LT peaks at the beginning and the end of the evaporation. At the beginning of the thinfilm deposition, the $\beta$ peak at $q_{\|}=1.38 \AA^{-1}$ intensity was stronger in intensity than the $(020)$ LT peak $\left(q_{\|} \approx 1.6 \AA^{-1}\right)$. After the full $50 \mathrm{~nm}$ film growth, the intensity of the $\beta$ peak was notably less intense than its maximum, while the (020) LT peak intensity was strongest. Additionally, a weak LT (011) peak at $q_{\|}=1.32 \AA^{-1}$ was detected, which was not present in the early stages of the $\alpha-6 \mathrm{~T}$ deposition. A timelapse of the full scattering images is available in the electronic Supporting Information. The peaks were fitted as described above to calculate the scattering intensity and the full width halfmaximum (FWHM). The size of the islands was estimated according to the Scherrer equation. Using this equation, the grain size, i.e., the diameter of the growing polymorph grains, can be plotted against the film thickness. The results, plotted in Figure 5B, show that after an initial increase in the grain size during the early stages of the film growth, the domains of both the $\beta$ - and LT-phases contracted in size until a film thickness of $\approx 19 \mathrm{~nm}$ where the LT-phase reached an equilibrium grain size value of $\approx 15 \mathrm{~nm}$ and the $\beta$-phase continued to shrink to a final grain size of $\approx 10 \mathrm{~nm}$ until the deposition was stopped. Finally, the integrated scattering intensities (ISI) of the $\beta$ peak and the LT (020) peak were normalized to the maximum value (LT ISI at the end of the film growth). The normalized ISI can then be used to compare the scattering intensity of the $\alpha$-6T-phases throughout the film growth (Figure 5C).

From the ISI data, three regions can be distinguished: region (1), where the $\beta$ - and LT-phase ISI increase quickly, and the $\beta$ phase ISI dominates; (2), where the rate of ISI increase slows for both the $\beta$ - and the LT-phases; and (3), where the LTphase ISI again increases while the $\beta$-phase ISI remains relatively constant. The first region of rapid ISI increase occurs up to about $4.8 \mathrm{~nm}(2 \mathrm{ML})$, roughly corresponding to the region of monolayer growth. The ISI then slows at the beginning of region 2, which occurs at the onset of island growth up to a thickness of approximately $8 \mathrm{~nm}$. Region 3 then begins at a film thickness of $\approx 8 \mathrm{~nm}$, and it is at this point that the ISI of the LT-phase continues to increase while the $\beta$-phase ISI stagnates until the deposition is stopped at $50 \mathrm{~nm}$ and the ISI of the LT-phase is more than twice that of the $\beta$-phase. Analyses of the $\beta$ - and LT-phase peak positions (Figures S2 and 6 , respectively) revealed that the start of region 3 corresponded with a sudden increase in the LT-phase (020) peak spacing, as seen in Figure 6, near a thickness of $8 \mathrm{~nm}$. This abrupt shift in $d$-spacing is indicative of strain relief, which accompanied the transition from monolayer to island growth associated with the SK growth mode. However, the GISAXS data indicated that the island growth occurred from a thickness of approximately $4 \mathrm{~nm}$, which corresponds to the start of region 2 . This finding suggests that although the island growth found in the GISAXS and AFM data occurs at smaller thicknesses, the full extent of the lattice strain induced by the glass surface is not relieved until a thickness of $8 \mathrm{~nm}$. Indeed, this is the thickness in which the off-specular peak intensities completely vanish. In the intermediate growth region (2), the growth of two polymorphs stagnates until the lattice strain is relaxed, at which point the LT-phase grows notably quicker than the $\beta$-phase. In contrast to the relaxing of the $\alpha-6 \mathrm{~T} \mathrm{LT}$ phase, the $d$-spacing of the $\beta$-phase peak did not undergo any substantial shifts following the growth mode (Figure S3) shift.

The in situ GIWAXS results obtained here closely mirror those reported by Lorch et al., ${ }^{26}$ who reported the growth of competing $\alpha-6 \mathrm{~T}$ crystal phases on $\mathrm{SiO}_{2}$ substrates at both 308 and $373 \mathrm{~K}$. However, our ambient temperature measurements on glass more closely resemble the growth they reported for higher substrate temperatures $(373 \mathrm{~K})$, where the $\beta$-phase dominated in the early stages of growth, after which the LTphase began to dominate. They reported that at high temperature, at thicknesses above $7 \mathrm{~nm}$, the LT-phase takes over the $\beta$-phase, nearly equal to the $8 \mathrm{~nm}$ thickness where we observed the $\beta$ - to LT-phase transition. Conversely, on $\mathrm{SiO}_{2}$ at $308 \mathrm{~K}$, a temperature similar to our ambient measurements on glass substrates, their results showed that the $\beta$-phase dominates throughout the deposition process. Furthermore, though the measurements by Lorch et al. show a larger LTphase $d$-spacing for the films grown at high temperature, the 

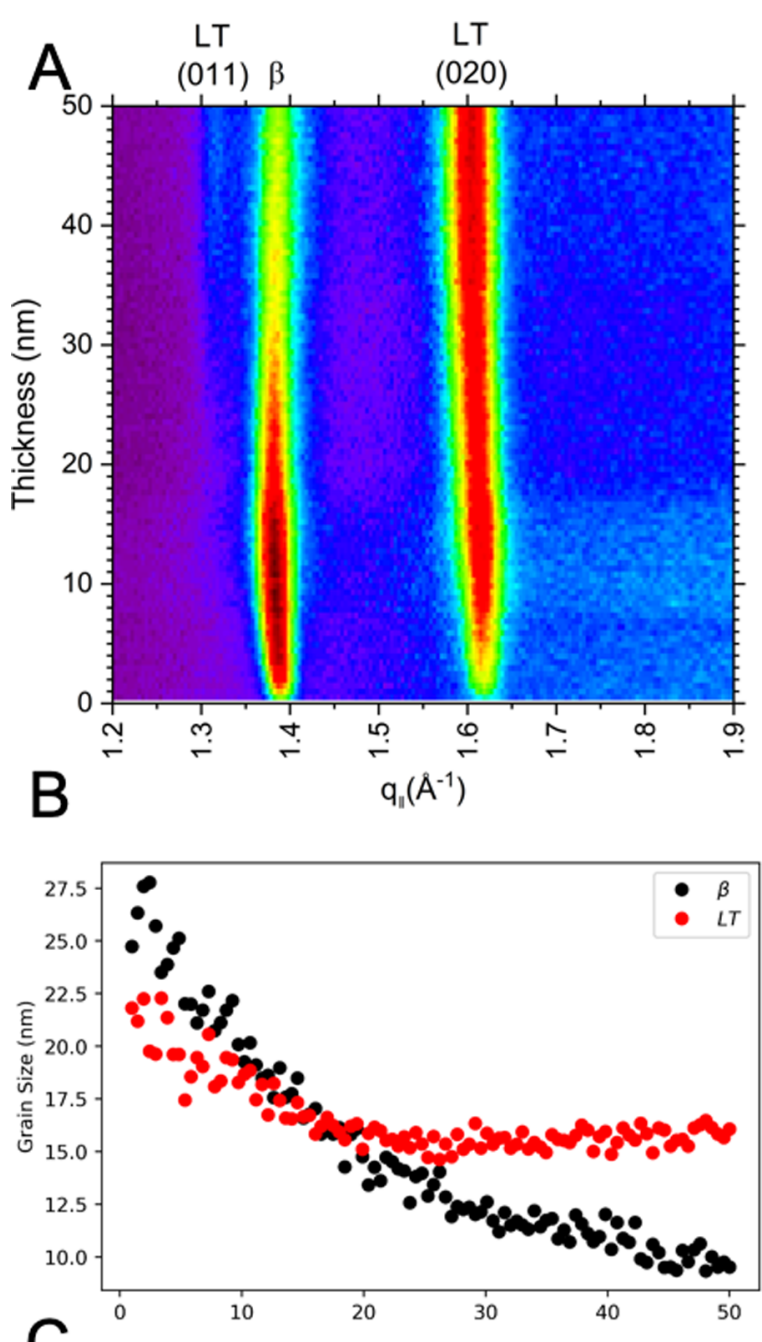

$\mathrm{C}$

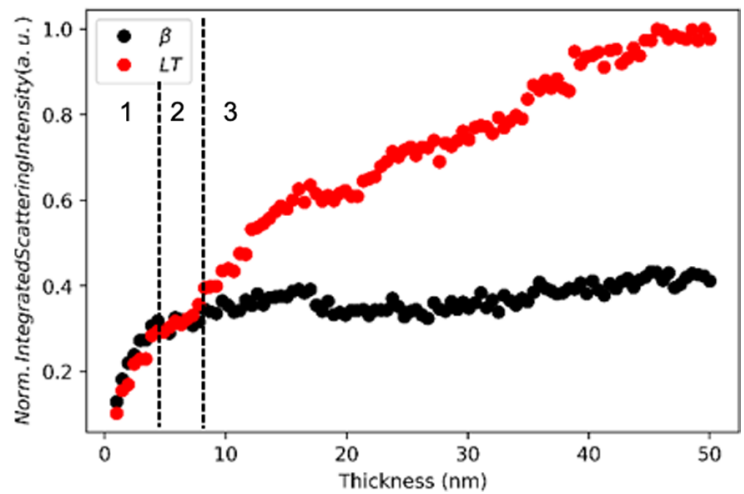

Figure 5. Real-time GIWAXS data of $\alpha-6 \mathrm{~T}$ deposited on glass at room temperature. (A) Real-time GIWAXS scans of vacuumdeposited $\alpha$-6T showing the evolution of the LT- and $\beta$-phases during the film growth. (B) Real-time evolution of the $\alpha$-6T $\beta$-phase and LT-phase (020) grain sizes. (C) Normalized integrated scattering intensity, with dashed lines indicating the regions of rapid $\beta$-phase growth (1), slowed growth (2), and LT-phase dominated growth (3).

real-time GIWAXS results at both high and low temperatures do not indicate surface strain such as we have observed on glass at ambient temperature. This disparity highlights the strain-mediated templating potential of the substrate on which $\alpha-6 \mathrm{~T}$ is deposited, a key consideration in device fabrication,



Figure 6. Real-time evolution of the $\alpha$-6T (020)-LT peak position (black) and $d$-spacing during film deposition.

given the distinct physical properties, such as absorption ${ }^{27}$ displayed by each $\alpha-6 \mathrm{~T}$ polymorph.

\section{CONCLUSIONS}

Through the use of real-time in situ X-ray scattering experiments, we were able to directly probe the growth mode of $\alpha-6 \mathrm{~T}$ on glass at room temperature from the molecular scale to $\approx 200 \mathrm{~nm}$. We employed in situ GISAXS to probe the formation and morphology of nanoscale aggregates, and in situ GIWAXS to probe the evolution of the crystalline structure of the growing films, both in real time. The GISAXS data revealed that $\alpha-6 \mathrm{~T}$ films grow according to a StranskiKrastanov growth mode where two-dimensional (2D) layerby-layer growth transitioned to three-dimensional (3D) island growth. The shift from $2 \mathrm{D}$ to $3 \mathrm{D}$ island growth was found to occur after two monolayers were deposited. The presence of both monolayer and island morphologies was confirmed through direct imaging via AFM. In situ GIWAXS measurements revealed the changing crystalline structure of the thin film, where initially more of the $\alpha$-6T $\beta$-phase formed on the glass surface, but when the film thickness exceeded $\approx 8 \mathrm{~nm}$, the LT-phase began to dominate. Taken together, the results present a multi length scale characterization of the evolution of vacuum-deposited $\alpha-6 \mathrm{~T}$ films on weakly interacting substrates, revealing a growth mode where both the molecular scale and the nanoscale morphology undergo a transition from surface to bulk-mediated growth. In addition to emphasizing the capability of the combination of in situ GIWAXS and GISAXS to characterize the thin-film growth, the findings highlight the importance of the surface in controlling the morphology and crystalline structure of vacuum-deposited films. Further in situ studies on additional OSC-relevant substrates and the characterization of device parameters will aid in the development of structure-property relationships necessary to improve performance.

\section{ASSOCIATED CONTENT}

SI Supporting Information

The Supporting Information is available free of charge at https://pubs.acs.org/doi/10.1021/acs.jpcc.0c00447.

Simulation model and parameters; comparison of experimental and simulated off specular scattering peaks; real time evolution of the $\alpha$-6T $\beta$-phase peak 
position and $d$-spacing during film deposition; AFM (PDF)

Real-time GISAXS timelapse (AVI)

Real-time GIWAXS timelapse (AVI)

\section{AUTHOR INFORMATION}

\section{Corresponding Authors}

T. L. Derrien - Diamond Light Source, Didcot, Oxfordshire OX11 ODE, United Kingdom; 다이.org/0000-0001-78752278; Email: thomas.derrien@diamond.ac.uk

M. Riede - Clarendon Laboratory, Department of Physics, University of Oxford, Oxford, Oxfordshire OX1 3PU, United Kingdom; (1) orcid.org/0000-0002-5399-5510;

Email: moritz.riede@physics.ox.ac.uk

\section{Authors}

A. E. Lauritzen - Clarendon Laboratory, Department of Physics, University of Oxford, Oxford, Oxfordshire OX1 3PU, United Kingdom

P. Kaienburg - Clarendon Laboratory, Department of Physics, University of Oxford, Oxford, Oxfordshire OX1 3PU, United Kingdom

J. F. M. Hardigree - Clarendon Laboratory, Department of Physics, University of Oxford, Oxford, Oxfordshire OX1 3PU, United Kingdom

C. Nicklin - Diamond Light Source, Didcot, Oxfordshire OX11 ODE, United Kingdom

Complete contact information is available at:

https://pubs.acs.org/10.1021/acs.jpcc.0c00447

\section{Notes}

The authors declare no competing financial interest.

\section{ACKNOWLEDGMENTS}

T.L.D. prepared the original manuscript of the draft with all authors contributing to the reviewing and editing process. T.L.D., A.E.L., P.K., and J.F.M. performed the experiments and T.L.D. analyzed the data. The project was conceived by T.L.D., A.E.L., C.N., and M.R., with C.N. and M.R. providing supervision. T.L.D., P.K., C.N., and M.R. acknowledge funding for this work from the UKRI-GCRF grant Synchrotron Techniques for African Research and Technology (START) ST/R002754/1. A.E.L. thanks EPSRC for funding through the Doctoral Training Partnership (EP/N509711/1) as well as STFC and the ISIS Neutron and Muon facility and project (1948713). Access to Diamond beamtime at I07 was provided under experiment Nos. SI20426-1 and NT24871-1. The authors thank A. Warne, J. Rawle, H. Hussain, and F. Carla (Diamond Light Source) for their assistance with beamline instrumentation. They are grateful to J. Naylor, D. Wicks, and A. Dorman of K. J. Lesker Ltd. for generously providing deposition control and evaporation sources along with technical support for MINERVA, which was the result of an STFC CLASP project (ST/L006294/1).

\section{REFERENCES}

(1) Warren, P. R.; Hardigree, J. F. M.; Lauritzen, A. E.; Nelson, J.; Riede, M. Tuning the Ambipolar Behaviour of Organic Field Effect Transistors via Band Engineering. AIP Adv. 2019, 9, No. 035202.

(2) Torsi, L.; Magliulo, M.; Manoli, K.; Palazzo, G. Organic FieldEffect Transistor Sensors: a Tutorial Review. Chem. Soc. Rev. 2013, $42,8612-8628$.
(3) Di, D.; et al. High-Performance Light-Emitting Diodes Based on Carbene-Metal-Amides. Science 2017, 356, 159-163.

(4) Ramirez, I.; Causa', M.; Zhong, Y.; Banerii, N.; Riede, M. Key Tradeoffs Limiting the Performance of Organic Photovoltaics. Adv. Energy Mater. 2018, 8, No. 1703551.

(5) Inganäs, O. Organic Photovoltaics over Three Decades. Adv. Mater. 2018, 30, No. 1800388.

(6) Heliatek, G. https://www.heliatek.com/about/.

(7) Ziehlke, H.; Fitzner, R.; Koerner, C.; Gresser, R.; Reinold, E.; Buerle, P.; Leo, K.; Riede, M. K. Side Chain Variations on a Series of Dicyanovinyl-Terthiophenes: A Photoinduced Absorption Study. J. Phys. Chem. A 2011, 115, 8437-8446.

(8) Schwarze, M.; et al. Band Structure Engineering in Organic Semiconductors. Science 2016, 352, 1446-1449.

(9) Sharifzadeh, S.; Wong, C. Y.; Wu, H.; Cotts, B. L.; Kronik, L.; Ginsberg, N. S.; Neaton, J. B. Relating the Physical Structure and Optoelectronic Function of Crystalline TIPS-Pentacene. Adv. Funct. Mater. 2015, 25, 2038-2046.

(10) Brigeman, A. N.; Fusella, M. A.; Yan, Y.; Purdum, G. E.; Loo, Y.-L.; Rand, B. P.; Giebink, N. C. Revealing the Full Charge Transfer State Absorption Spectrum of Organic Solar Cells. Adv. Energy Mater. 2016, 6, No. 1601001.

(11) Lunt, R. R.; Benziger, J. B.; Forrest, S. R. Relationship Between Crystalline Order and Exciton Diffusion Length in Molecular Organic Semiconductors. Adv. Mater. 2010, 22, 1233-1236.

(12) Mikhnenko, O. V.; Blom, P. W. M.; Nguyen, T.-Q. Exciton Diffusion in Organic Semiconductors. Energy Environ. Sci. 2015, 8, $1867-1888$.

(13) Loi, M. A.; da Como, E.; Dinelli, F.; Murgia, M.; Zamboni, R.; Biscarini, F.; Muccini, M. Supramolecular Organization in Ultra-Thin Films of -Sexithiophene on Silicon Dioxide. Nat. Mater. 2004, 4, 8185 .

(14) Purdum, G. E.; Yao, N.; Woll, A.; Gessner, T.; Weitz, R. T.; Loo, Y.-L. Understanding Polymorph Transformations in CoreChlorinated Naphthalene Diimides and their Impact on Thin-Film Transistor Performance. Adv. Funct. Mater. 2016, 26, 2357-2364.

(15) Jurchescu, O. D.; Mourey, D. A.; Subramanian, S.; Parkin, S. R.; Vogel, B. M.; Anthony, J. E.; Jackson, T. N.; Gundlach, D. J. Effects of Polymorphism on Charge Transport in Organic Semiconductors. Phys. Rev. B 2009, 80, No. 085201.

(16) Kan, Z.; Colella, L.; Canesi, E. V.; Vorobiev, A.; Skrypnychuk, V.; Terraneo, G.; Barbero, D. R.; Bertarelli, C.; MacKenzie, R. C. I.; Keivanidis, P. E. Charge transport control via polymer polymorph modulation in ternary organic photovoltaic composites. J. Mater. Chem. A 2016, 4, 1195-1201.

(17) Matsushima, T.; Adachi, C. Extremely Low Voltage Organic Light-emitting Diodes with p-doped Alpha-Sexithiophene Hole Transport and n-doped Phenyldipyrenylphosphine Oxide Electron Transport Layers. Appl. Phys. Lett. 2006, 89, No. 253506.

(18) Dinelli, F.; Murgia, M.; Levy, P.; Cavallini, M.; Biscarini, F.; de Leeuw, D. M. Spatially Correlated Charge Transport in Organic Thin Film Transistors. Phys. Rev. Lett. 2004, 92, No. 116802.

(19) Taima, T.; Shahiduzzaman, M.; Ishizeki, T.; Yamamoto, K.; Karakawa, M.; Kuwabara, T.; Takahashi, K. Sexithiophene-Based Photovoltaic Cells with High Light Absorption Coefficient via Crystalline Polymorph Control. J. Phys. Chem. C 2017, 121, 19699-19704.

(20) Veenstra, S. C.; Malliaras, G. G.; Brouwer, H. J.; Esselink, F. J.; Krasnikov, V. V.; van Hutten, P. F.; Wildeman, J.; Jonkman, H. T.; Sawatzky, G. A.; Hadziioannou, G. Sexithiophene-C60 Blends as Model Systems for Photovoltaic Devices. Synth. Met. 1997, 84, 971972.

(21) Duva, G.; et al. Thin-Film Texture and Optical Properties of Donor/Acceptor Complexes. Diindenoperylene/F6TCNNQ vs Alpha-Sexithiophene/F6TCNNQ. J. Phys. Chem. C 2018, 122, 18705-18714.

(22) Lorcy, D.; Cava, M. P. Poly(isothianaphthenebithiophefe): A New Regularly Structured Polythiophene Analogue. Adv. Mater. 1992, 4, 562-564. 
(23) Destri, S.; Mascherpa, M.; Porzio, W. Mesophase Formation in -Sexithienyl at High Temperature An X-ray Diffraction Study. Adv. Mater. 1993, 5, 43-45.

(24) Horowitz, G.; Bachet, B.; Yassar, A.; Lang, P.; Demanze, F.; Fave, J.-L.; Garnier, F. Growth and Characterization of Sexithiophene Single Crystals. Chem. Mater. 1995, 7, 1337-1341.

(25) Siegrist, T.; Fleming, R.; Haddon, R.; Laudise, R.; Lovinger, A.; Katz, H.; Bridenbaugh, P.; Davis, D. The crystal Structure of the High-Temperature Polymorph of Hexathienyl (6T/HT). J. Mater. Res. 1995, 10, 2170-2173.

(26) Lorch, C.; Banerjee, R.; Frank, C.; Dieterle, J.; Hinderhofer, A.; Gerlach, A.; Schreiber, F. Growth of Competing Crystal Phases of -Sexithiophene Studied by Real-Time in Situ X-ray Scattering. J. Phys. Chem. C 2015, 119, 819-825.

(27) Pithan, L.; Cocchi, C.; Zschiesche, H.; Weber, C.; Zykov, A.; Bommel, S.; Leake, S. J.; Schäfer, P.; Draxl, C.; Kowarik, S. Light Controls Polymorphism in Thin Films of Sexithiophene. Cryst. Growth Des. 2015, 15, 1319-1324.

(28) Klett, B.; Cocchi, C.; Pithan, L.; Kowarik, S.; Draxl, C. Polymorphism in -Sexithiophene Crystals: Relative Stability and Transition Path. Phys. Chem. Chem. Phys. 2016, 18, 14603-14609.

(29) Servet, B.; Horowitz, G.; Ries, S.; Lagorsse, O.; Alnot, P.; Yassar, A.; Deloffre, F.; Srivastava, P.; Hajlaoui, R. Polymorphism and Charge Transport in Vacuum-Evaporated Sexithiophene Films. Chem. Mater. 1994, 6, 1809-1815.

(30) Moser, A.; et al. A Disordered Layered Phase in Thin Films of Sexithiophene. Chem. Phys. Lett. 2013, 574, 51-55.

(31) Lorch, C.; Broch, K.; Belova, V.; Duva, G.; Hinderhofer, A.; Gerlach, A.; Jankowski, M.; Schreiber, F. Growth and Annealing Kinetics of $\alpha$-Sexithiophene and Fullerene $\mathrm{C}_{6} 0$ Mixed Films. J. Appl. Crystallogr. 2016, 49, 1266-1275.

(32) Nicklin, C.; Martinez-Hardigree, J.; Warne, A.; Green, S.; Burt, M.; Naylor, J.; Dorman, A.; Wicks, D.; Din, S.; Riede, M. MINERVA: A facility to study Microstructure and INterface Evolution in Realtime under VAcuum. Rev. Sci. Instrum. 2017, 88, 103901.

(33) Filik, J.; et al. Processing Two-Dimensional X-ray Diffraction and Small-Angle Scattering Data in DAWN 2. J. Appl. Crystallogr. 2017, 50, 959-966.

(34) Nečas, D.; Klapetek, P. Gwyddion: An Open-Source Software for SPM Data Analysis. Open Phys. 2012, 10, 181-188.

(35) Kowarik, S. Thin Film Growth Studies Using Time-Resolved Xray Scattering. J. Phys.: Condens. Matter 2016, 29, No. 043003.

(36) Pospelov, G.; Van Herck, W.; Burle, J.; Carmona Loaiza, J. M.; Durniak, C.; Fisher, J. M.; Ganeva, M.; Yurov, D.; Wuttke, J. BornAgain: Software for Simulating and Fitting Grazing-Incidence Small-Angle Scattering. J. Appl. Crystallogr. 2020, 53, 262-276.

(37) Stranski, I. N.; Krastanow, L. Zur Theorie der Orientierten Ausscheidung von Ionenkristallen Aufeinander. Monatsh. Chem. 1937, $71,351-364$.

(38) Sun, L.; Berkebile, S.; Weidlinger, G.; Denk, M.; Denk, R.; Hohage, M.; Koller, G.; Netzer, F. P.; Ramsey, M. G.; Zeppenfeld, P. Layer Resolved Evolution of the Optical Properties of $\alpha$ Sexithiophene Thin Films. Phys. Chem. Chem. Phys. 2012, 14, 13651-13655.

(39) Ghanbari, E.; Wagner, T.; Zeppenfeld, P. Layer-Resolved Evolution of Organic Thin Films Monitored by Photoelectron Emission Microscopy and Optical Reflectance Spectroscopy. J. Phys. Chem. C 2015, 119, 24174-24181.

(40) Scherrer, P. Bestimmung der Gre und der Inneren Struktur von Kolloidteilchen Mittels Rntgenstrahlen. Kolloidchem. Lehrb. 1912, 387-409.

(41) Smilgies, D.-M. Scherrer Grain-Size Analysis Adapted to Grazing-Incidence Scattering With Area Detectors. J. Appl. Crystallogr. 2009, 42, 1030-1034. 